\title{
Topological quantum memory interfacing atomic and superconducting qubits
}

\author{
Zheng-Yuan Xue, ${ }^{1}$ Zhang-qi Yin,, \& Yan Chen, ${ }^{3}$ Z. D. Wang,, , O and Shi-Liang Zhu ${ }^{5}$, 团 \\ ${ }^{1}$ Guangdong Provincial Key Laboratory of Quantum Engineering and Quantum Materials, and School of Physics \\ and Telecommunication Engineering, South China Normal University, Guangzhou 510006, China \\ ${ }^{2}$ Center for Quantum Information, Institute of Interdisciplinary Information Science, Tsinghua University, Beijing 100084, China \\ ${ }^{3}$ Department of Physics, State Key Laboratory of Surface Physics and Laboratory of Advanced Materials, \\ Fudan University, Shanghai 200433, China \\ ${ }^{4}$ Department of Physics and Center of Theoretical and Computational Physics, The University of Hong Kong, \\ Pokfulam Road, Hong Kong, China \\ ${ }^{5}$ National Laboratory of Solid State Microstructure and Department of Physics, Nanjing University, Nanjing 210093, China
}

(Dated: August 9, 2021)

\begin{abstract}
We propose a scheme to manipulate a topological spin qubit which is realized with cold atoms in a onedimensional optical lattice. In particular, by introducing a quantum opto-electro-mechanical interface, we are able to first transfer a superconducting qubit state to an atomic qubit state and then to store it into the topological spin qubit. In this way, an efficient topological quantum memory could be constructed for the superconducting qubit. Therefore, we can consolidate the advantages of both the noise resistance of the topological qubits and the scalability of the superconducting qubits in this hybrid architecture.

PACS numbers: 03.67.Lx, 42.50.Dv, 07.10.Cm
\end{abstract}

\section{INTRODUCTION}

Quantum computation has attracted much attention as it is able to solve diverse classes of hard problems. Superconducting circuits are promising for implementing quantum computer hardwares as they are potentially scalable [1]. As a superconducting qubit is usually quite sensitive to the external environments and background noises, its coherence time is generally rather short [2]. A promising strategy out of this difficulty is based on the topological idea [3]: a topological qubit is largely insensitive to major sources of local noises, and thus can be used to form an efficient topological quantum memory (TQM).

Recently, with the potential applications in topological quantum computation, topological states of matter have attracted renewed interests [4-6]. In particular, time-reversal invariant topological insulators [7-11] have been reported experimentally, and thus have greatly stimulated the study of topological phases [4]. In engineering topological phases, the spin-orbit (SO) interaction usually plays an important role. Therefore, with recent great achievements in realizing artificial SO interaction in cold atom system [12-18], it becomes a new platform to probe topological phases in a fully controllable way [19]. Recently, Liu et al. [20] proposed to observe and manipulate topological edge spins realized in a onedimensional (1D) optical lattice with experimentally realized SO interaction. The nontrivial topology there supports two degenerate zero modes, which are topologically stable, and thus can be used to construct a topological spin qubit (TSQ).

Here, we propose a scheme to realize an interface between this TSQ and a solid-state superconducting qubit. This hybrid system may allow us to combine the advantages of both the

\footnotetext{
* yinzhangqi@gmail.com

† zwang@hku.hk

$\ddagger$ slzhu@nju.edu.cn
}

noise resistance of the topological qubits and the scalability of the superconducting qubits. With the help of cavity assisted interaction [21], we show that local operations can be implemented for the TSQ. Our particular interest lies in using this TSQ as a TQM, where we can store quantum information of both atomic and superconducting qubits. Meanwhile, recent experiments shown that the TSQ was not only a theoretical proposal, but also within experimental feasibility [22]. Finally, we note that there are proposals considering the hybrid systems consisting of superconducting qubits and nitrogenvacancy centers. However, as the coupling between a single nitrogen-vacancy center and a cavity is usually very weak, below $0.1 \mathrm{kHz}$ level [23], previous works mainly focus on using ensemble of nitrogen-vacancy centers [23-26], where the dephasing time of the ensemble is much shorter comparing with a single nitrogen-vacancy center.

To store the state of a superconducting qubit [blue rectangle in Fig. 1(a)] into the TSQ formed by the atomic lattice, as shown in Fig. 1(a), we firstly transfer the state of the superconducting qubit to an ancillary atomic qubit [pink circle in Fig. 1(a)] and then store it into the TSQ. As the superconducting and ancillary atomic qubits are of vastly different frequencies, a quantum opto-electro-mechanical setup is needed, where a mechanical oscillator mediates the coherent coupling of both microwave and optical photons [27-31]. It was reported that this interface had been realized in recent experiments [27, 28]. The superconducting qubit interacts with a microwave cavity mode in a circuit QED scenario [32], while the atoms interact with the optical cavity mode. When the intra-cavity interaction is switched on, one can obtain high fidelity state transfer from a superconducting qubit to the ancillary atomic qubit. By switching off the intra-cavity interaction, we show that a TQM for the ancillary atom can be constructed. Combining the two processes become particularly arresting as we can store a superconducting qubit state into the TQM, which provides an alternation for interfacing topological and superconducting qubits [33-36]. 
(a)
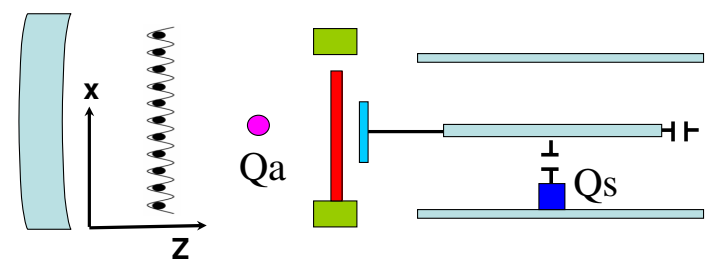

(b)
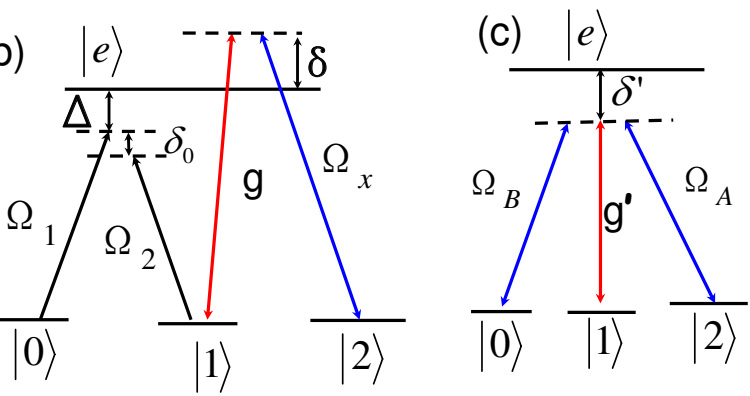

FIG. 1. Illustration of our scheme. (a) A hybrid architecture consists of two-cavity optomechanical system. A mechanical oscillator (red) mediates the coupling of the optical (left) and microwave cavities (right). A 1D optical lattice, constructing our TQM, is inside the optical cavity. An ancillary atom (pink circle) is incorporated to engineer the cavity photon state and its information can be stored in the TQM. (b) The level structure of atoms in the optical lattice. The transitions $|0\rangle \rightarrow|e\rangle$ and $|1\rangle \rightarrow|e\rangle$ are induced by the lasers in a large one-photon detuning $|\Delta| \gg \Omega$ and a small two-photon detuning $\left|\delta_{0}\right| \ll \Omega$. The transition $|1\rangle \rightarrow|e\rangle$ is dispersively coupled to the cavity field to achieve the QND Hamiltonian. To obtain effective switch of the cavity-assisted interaction, a strong control laser of Rabi frequency $\Omega_{x}$ driving the transition of $|2\rangle \rightarrow|e\rangle$ is also introduced. (c) The level structure of the ancillary atom.

\section{A TOPOLOGICAL SPIN QUBIT}

The TSQ is based on a quasi-1D cold fermions with threelevel Lamda configuration trapped in an optical lattice [20]. As shown in Fig. 1(b), the transitions $|0\rangle \rightarrow|e\rangle$ and $|1\rangle \rightarrow$ $|e\rangle$ are induced by the lasers, with Rabi-frequencies $\Omega_{1}=$ $\Omega \sin \left(k_{0} x / 2\right)$ and $\Omega_{2}=\Omega \cos \left(k_{0} x / 2\right)$, in a large one-photon detuning $|\Delta| \gg \Omega$ and a small two-photon detuning $\left|\delta_{0}\right| \ll$ $\Omega$, which is equivalent to a Zeeman field along the $z$ axis $\Gamma_{z}=$ $\hbar \delta_{0} / 2$ and can be precisely controlled. Then, adiabatically eliminating the excited state $|e\rangle$ yields the following effective Hamiltonian

$$
\begin{aligned}
H_{\mathrm{eff}}=\frac{p_{x}^{2}}{2 m}+\sum_{\sigma=0,1}\left[V_{\sigma}(x)+\Gamma_{z} \sigma_{z}\right]|\sigma\rangle\langle\sigma| \\
-[M(x)|0\rangle\langle 1|+\text { H.c. }],
\end{aligned}
$$

where $M(x)=M_{0} \sin \left(k_{0} x\right)$ with $M_{0}=\frac{\hbar \Omega^{2}}{2 \Delta}$ being an additional laser-induced Zeeman field along $x$ axis. To form the 1D lattice, the optical dipole trapping potentials are chosen as $V_{\sigma}(x)=-V_{0} \cos ^{2}\left(k_{0} x\right)$ with the lattice trapping frequency being $\omega=\left(2 V_{0} k_{0}^{2} / m\right)^{1 / 2}[19]$. The states $|0\rangle$ and $|1\rangle$ are defined by spin up and down of a pseudo-spin, respectively. The tight-binding description of Hamiltonian (11) is defined as the case when the fermions occupy the lowest $s$-orbitals $\phi_{s \sigma}$. Redefining the spin-down operator as $\hat{c}_{j \downarrow} \rightarrow e^{i \pi x_{j} / a} \hat{c}_{j \downarrow}$ with $a$ being lattice constant, the tight-binding Hamiltonian reads [20]

$$
\begin{aligned}
H= & -t_{s} \sum_{<i, j>}\left(\hat{c}_{i \uparrow}^{\dagger} \hat{c}_{j \uparrow}-\hat{c}_{i \downarrow}^{\dagger} \hat{c}_{j \downarrow}\right)+\sum_{i} \Gamma_{z}\left(\hat{n}_{i \uparrow}-\hat{n}_{i \downarrow}\right) \\
& +\left[\sum_{j} t_{\mathrm{so}}^{(0)}\left(\hat{c}_{j \uparrow}^{\dagger} \hat{c}_{j+1 \downarrow}-\hat{c}_{j \uparrow}^{\dagger} \hat{c}_{j-1 \downarrow}\right)+\text { H.c. }\right]
\end{aligned}
$$

where $\hat{n}_{i \sigma}=\hat{c}_{i \sigma}^{\dagger} \hat{c}_{i \sigma}$,

$$
\begin{aligned}
t_{s} & =\int d x \phi_{s \sigma}^{(j)}(x)\left[\frac{p_{x}^{2}}{2 m}+V\right] \phi_{s \sigma}^{(j+1)}(x), \\
t_{\mathrm{so}}^{(0)} & =\frac{\Omega_{0}^{2}}{\Delta} \int d x \phi_{s}(x) \sin \left(2 k_{0} x\right) \phi_{s}(x-a) .
\end{aligned}
$$

In $k$ space, the tight-binding Hamiltonian can be rewritten as

$$
H_{k}=-\sum_{k, \sigma \sigma^{\prime}} \hat{c}_{k, \sigma}^{\dagger}\left[d_{z}(k) \sigma_{z}+d_{y}(k) \sigma_{y}\right]_{\sigma, \sigma^{\prime}} \hat{c}_{k, \sigma^{\prime}},
$$

where $d_{y}=2 t_{\mathrm{so}}^{(0)} \sin (k a)$ and $d_{z}=-\Gamma_{z}+2 t_{s} \cos (k a)$. This Hamiltonian describes a nontrivial topological insulator when $\left|\Gamma_{z}\right|<2 t_{s}$ and otherwise a trivial insulator, with a bulk gap $E_{g}=\min \left\{\left|2 t_{s}-\right| \Gamma_{z}||, 2\left|t_{\mathrm{so}}^{(0)}\right|\right\}$. The nontrivial topology supports two degenerate boundary modes, and each mode equals one-half of a spin $1 / 2$ particle, similar to the relation between a Majorana fermion and a complex fermion in topological superconductors [37]. The zero modes are robust to local noises, and thus may form a TSQ which we can use as a TQM. The TSQ can also be obtained in the middle of the lattice by creating mass domain. Local operations upon the TSQ can be achieved by applying a local Zeeman term $\mathbf{B}_{y}=\Gamma_{0} \sigma_{y}$ or $\mathbf{B}_{z}=\Gamma_{0} \sigma_{z}$ [20]. When $\left|\Gamma_{0}\right|>2\left|t_{\mathrm{so}}^{(0)}\right|$ a mass domain is created, associated with two midgap spin states $\left|\psi_{ \pm}\right\rangle$localized around the two edges of the TSQ. Let $\left|\psi_{+}\right\rangle$be initially occupied, reducing $\left|\Gamma_{0}\right|$ smoothly can open the coupling in $\left|\psi_{ \pm}\right\rangle$ and lead the TSQ state to evolve. If we apply the Zeeman field along $z(y)$ axis, the TSQ evolves in the $x-y(x-z)$ plane [20].

\section{TQM FOR AN ATOMIC QUBIT}

We then show how to store the state of an ancillary atom into the TSQ. For our TQM purpose, we need controlled manipulation of the TSQ, and thus a controllable local Zeeman field. Here, we propose to implement this controlled manipulation with cavity-assisted quantum nondemolition (QND) Hamiltonian [21]. To achieve this, we introduce another level, and thus the fermions are now in four-level Tripod configuration. For ${ }^{6} \mathrm{Li}$ and ${ }^{40} \mathrm{~K}$ atoms, we can find appropriate hyperfine levels to meet the requirement. The coupling structure is shown in Fig. 1(b). To implement the QND Hamiltonian $H_{Q N D}=\chi a^{\dagger} a \sum_{l} \sigma_{l}^{z}$, we set the cavity mode to couple the 
transition of $|1\rangle \leftrightarrow|e\rangle$ with a strength $g$ and blue detuning $\delta$, where $\chi=g^{2} /(2 \delta)$ and $a\left(a^{\dagger}\right)$ is the annihilation (creation) operator for the cavity photon. As the cavity assisted interaction is always-on, if we want the interaction to act only on the atoms within certain area, we should be able to decouple the interaction outside the wanted area. This can be done by using another laser which couples the transition of excited state to another level with a Rabi frequency $\Omega_{x}$, i.e., $|2\rangle \leftrightarrow|e\rangle$, as shown in Fig. 1(b). When $\Omega_{x} \gg g$, the destructive interference of excitation pathways from the two transition ensures that the so-called dark state, which decoupled the atoms from interacting with both optical fields [38, 39]. This QND Hamiltonian preserves the photon number $n_{c}$ of the cavity mode. Within the $n_{c} \in\{0,1\}$ subspace, the evolution of the QND Hamiltonian for an interaction time of $\tau=\pi /(2 \chi)$ yields [21]

$$
U=\exp \left[-i \tau H_{Q N D}\right]=\left\{\begin{array}{cl}
\mathbf{I} & \text { for } n_{c}=0 \\
(-i)^{N} \prod_{l} \sigma_{l}^{z} & \text { for } n_{c}=1
\end{array}\right.
$$

where $N$ being the number of the selected atoms. If the cavity is initially prepared in the $n_{c}=1$ state, the global operation in Eq. (4) reduces to a string operation $U_{z}=\prod_{l} \sigma_{l}^{z}$. Note that all the string operators are equivalent to $U_{z}$ up to local single spin rotations [21]: $U_{x}=\prod_{l} \sigma_{l}^{x}=H U_{z} H$ and $U_{y}=$ $\prod_{l} \sigma_{l}^{y}=R U_{z} R$, where $H=\prod_{l} H_{l}$ and $R=\prod_{l} R_{l}$ with $H_{l}=\left(\sigma_{l}^{x}+\sigma_{l}^{z}\right) / \sqrt{2}$ being the Hadamard rotation and $R_{l}=$ $\exp \left(-i \frac{\pi}{4} \sigma_{l}^{z}\right)$. Therefore, as we can implement $U_{z}$, universal single qubit gates on the TSQ can be implemented.

When the cavity state is in a superposition state of $\mu|0\rangle_{c}+$ $\nu|1\rangle_{c}$, the global operation in Eq. (4) reduces to a controlledstring operation: $U_{1}=|0\rangle_{c}\langle 0|\otimes I+| 1\rangle_{c}\langle 1| \otimes U_{z}$. Here, we need to engineer the cavity photon states. However, it is actually easier to control an ancillary atom rather than to directly manipulate the photon number state. Therefore, we put an ancillary atom into the optical cavity, which is used to achieve controlled-string operations between the ancillary atom and the TSQ. The level structure of the ancillary atom is shown in Fig. 1(c), the transitions of $|1\rangle \leftrightarrow|e\rangle$ and $|2\rangle \leftrightarrow|e\rangle$ are coupled to the cavity field with strength $g^{\prime}$ and a laser with Rabi frequency $\Omega_{A}$, respectively. Meanwhile, the two couplings are in a two-photon resonance scenario with a red detuning $\delta^{\prime}$ to the exited state $|e\rangle$. Then, the two couplings are described by an effective Hamiltonian given as

$$
H_{e}=\lambda\left(a^{\dagger}|2\rangle\langle 1|+a| 1\rangle\langle 2|\right)
$$

where $\lambda=g^{\prime} \Omega_{A} / \delta^{\prime}$. To implement the controlled operations conditioned on the states of the ancillary atom, we choose the initial state as that the cavity mode is in a vacuum state $|0\rangle$ and the ancillary atom is in an arbitrary superposition state of $\alpha|0\rangle_{A}+\beta|1\rangle_{A}$. The procedure is listed as follows. (1) An interaction of $H_{e}$ for $t=\pi / \lambda$ coherently couples the cavity with the atom: $\alpha|0\rangle_{A}|0\rangle_{c}+\beta|2\rangle_{A}|1\rangle_{c}$. (2) The QND Hamiltonian for $\tau=\pi /(2 \chi)$ on the above intermediate state is applied. (3) Applying $H_{e}$ for another $t=\pi / \lambda$ will annihilate the cavity photon and restore the ancillary atom to its original state. In the steps (1) and (3), we have neglected a phase factor, which can be compensated by a sing-qubit rotation on the ancillary atom. In this way, one realizes a controlled-string operation conditioned on the state of the ancillary atom [21]

$$
U_{2}=|0\rangle_{A}\langle 0|\otimes I+| 1\rangle_{A}\langle 1| \otimes U_{z} .
$$

In particularly, upon local single spin rotations, one can obtain the controlled operations for the TSQ conditioned on the ancillary atom given as

$$
\begin{aligned}
& U_{c s}^{z}=|0\rangle_{A}\langle 0|\otimes I+| 1\rangle_{A}\langle 1| \otimes S_{\mathrm{TSQ}}^{z}, \\
& U_{c s}^{x}=|0\rangle_{A}\langle 0|\otimes I+| 1\rangle_{A}\langle 1| \otimes S_{\mathrm{TSQ}}^{x},
\end{aligned}
$$

where $S_{\mathrm{TSQ}}^{(x, z)}$ are the Pauli matrices for the TSQ.

With such controlled operations, one is able to access an efficient TQM [21]. For this purpose, we need a swap in gate defined as $U_{i n}=H_{A} U_{c s}^{z} H_{A} U_{c s}^{x}$ with $H_{A}$ being the Hadamard rotation on the ancillary atom, which swaps the ancillary atomic state $(\alpha|0\rangle+\beta|1\rangle)_{A}$ into the topological memory initialized in $\left|\psi_{+}\right\rangle$. For the inverse process, we need a swap out gate $U_{\text {out }}=U_{c s}^{x} H_{A} U_{c s}^{z} H_{A}$ that swaps the stored information back to the ancillary atom prepared in $|0\rangle_{A}$. The swap in (out) process corresponds to write (read) process for our TQM. The read process also provides us an alternative way of reading out the topological qubit, which is usually a hard problem.

We now turn to discuss the experimental feasibility of storing the ancillary atomic state into the TQM. (1) The TSQ considered here is robust in the large $N$ limit [37]. For finite $N$, the ground-state degeneracy is broken, and thus causes decoherence. However, the lifetime of the TSQ is exponentially increased with the increasing of $N$. Therefore, for small $N$, one may already obtain a relatively long coherence time for the TSQ [20]. (2) To implement the QND Hamiltonian, we use the large detuned scheme, which only requires large Purcell factor, i.e., $P=g^{2} /(\gamma \kappa)>1$ with $\gamma$ and $\kappa$ being the spontaneous decay rate of level $|e\rangle$ and the cavity decay rate, respectively. For our large detuned scheme, the effective spontaneous decay rate is suppressed to $\gamma_{e f f}=\gamma g^{2} / \delta^{2}$. Therefore, as the selected atoms decay independently, the total probability for photon loss is $P_{\text {loss }}^{N}=\left(\kappa+N \gamma_{\text {eff }}\right) \tau \geq 2 \pi \sqrt{N / P} \approx$ $3 \%$ for $N=5, g /(2 \pi)=220 \mathrm{MHz}$ [40], $\gamma=10 \mathrm{MHz}$, and $\kappa=1 \mathrm{MHz}$. (3) The addressing errors of the lasers are associated with a finite spread around the lattice points of atoms, which results in a tiny coupling between the addressing beam and the selected atoms, and thus leads to a finite lifetime for the level $|2\rangle$. The error probability associated with addressing each site is estimated to be $\varepsilon=1 \%$ [38, 39], which can be further suppressed. (4) The deviation of the QND interaction, which degrades the controlled string operation, can also be corrected by the quantum control techniques to arbitrarily high order [41]. (5) For the controlled string operations, we further need the light-atom interface, i.e., the reversible state transfer between light and the ancillary atom. With a strong lase field of $\Omega_{A}=1 \mathrm{GHz}$, the error rate can be achieved un$\operatorname{der} P_{i}=1 \%$. Finally, combining the above error channels, we may obtain a fidelity about $95 \%$ for a controlled operation. As both the read and write processes of the TQM need two controlled operations, a fidelity of $F_{1}=95 \% \times 95 \% \approx 90 \%$ can be obtained for the storage of the ancillary atomic state into the TQM. 


\section{INTERFACE WITH A SUPERCONDUCTING QUBIT}

We now show that by incorporating the above system with an additional quantum opto-electro-mechanics interface [27], we can achieve the storage of a superconducting qubit state into our TQM. The combined setup is shown in Fig. 1(a), where we consider the case of two-cavity optomechanical system. For our TQM purpose, we can firstly transfer the state of the superconducting qubit to the ancillary atomic qubit and then store the ancillary atomic qubit state into the TSQ as proposed previously. Therefore, we only need to consider the state transfer between the superconducting qubit and the ancillary atomic qubit in the following. This process is achieved by the quantum opto-electro-mechanical setup. In a circuit QED system, under the rotating-wave approximation, the interaction Hamiltonian takes the Jaynes-Cummings form [32]

$$
H_{m}=g_{m}\left(b^{\dagger} \sigma_{s}^{-}+b \sigma_{s}^{+}\right)
$$

where $g_{m}$ is the coupling strength of the superconducting qubit to the microwave cavity, the subscript "s" denotes that the operators belong to the superconducting qubit, $b$ and $b^{\dagger}$ are the annihilation and creation operators of the microwave cavity field, respectively. Similarly, the ancillary atom is also coupled to the optical cavity [40], as shown in Fig. 1(c), the transitions of $|1\rangle \leftrightarrow|e\rangle$ and $|0\rangle \leftrightarrow|e\rangle$ are coupled to the cavity field with strength $g^{\prime}$ and a laser with Rabi frequency $\Omega_{B}$, in the two-photon resonance way. Then, the two couplings are described by an effective Hamiltonian given as

$$
H_{o}=g_{o}\left(a^{\dagger}|0\rangle_{a}\langle 1|+a| 1\rangle_{a}\langle 0|\right)
$$

where $g_{o}=g^{\prime} \Omega_{B} / \delta^{\prime}$ and the subscript "a" stands for atom. Here, we consider that the opto-electro-mechanical coupling is enhanced by strongly driving of each cavity, resulting in an effective linear couplings [31]. Assuming that each cavity is far into the resolved-sideband regime and is driven near the red-detuned mechanical sideband, in the interaction picture, the interaction Hamiltonian reads

$$
H_{c}=G_{1}\left(d a^{\dagger}+d^{\dagger} a\right)+G_{2}\left(d b^{\dagger}+d^{\dagger} b\right),
$$

where $d$ and $d^{\dagger}$ are the annihilation and creation operators of the mechanical oscillator, respectively. The coupling between the mechanical resonator and cavity $i$ is denoted as $G_{i}$, which is controllable as they are proportional to the external driven amplitude, so we may choose $G_{1}=G_{2}=G$ for simplicity. The total Hamiltonian reads $H=H_{o}+H_{m}+H_{c}$, which conserves the total excitations and we restrict our discussions to be within the zero- and single-excitation subspaces. Transfer of the intra-cavity qubit states can be accomplished by modulating parameters of our system [42]. As the light-matter interaction is tunable, we can modulate $g_{o}=g_{m}=g$. The initial states of the ancillary atomic and superconducting qubits are assumed to be $|0\rangle_{a}$ and $\left|\psi_{s}\right\rangle=\left(\alpha|0\rangle_{s}+\beta|1\rangle_{s}\right)$, respectively. Deterministic quantum state transfer is realized when $\exp (-i t H)|000\rangle_{c}|0\rangle_{a}\left|\psi_{s}\right\rangle=|000\rangle_{c}\left|\psi_{a}\right\rangle|0\rangle_{s}$ is fulfilled, where $\left|\psi_{a}\right\rangle=\alpha|0\rangle_{a}+\beta|1\rangle_{a}$ and $|000\rangle_{c}$ means that the three bosonic modes are all in the vacuum states.

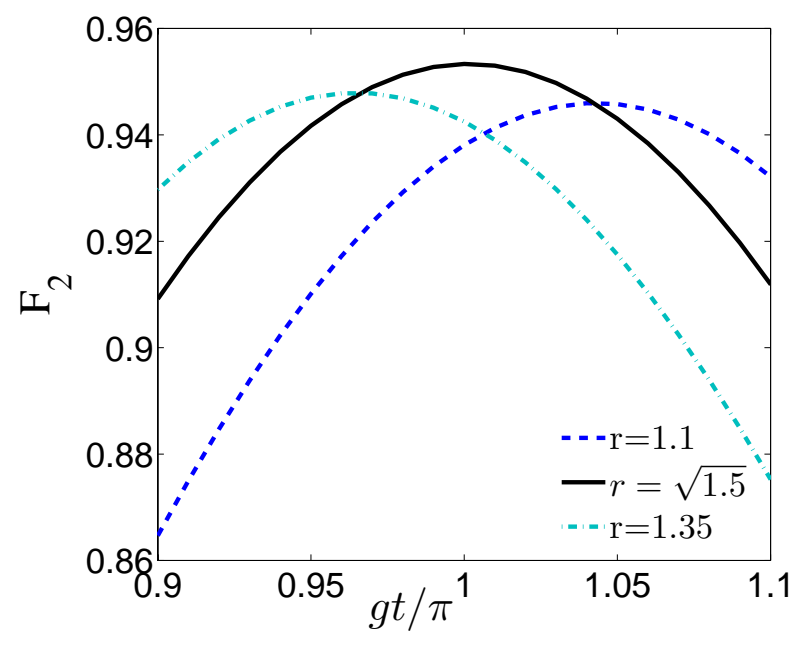

FIG. 2. The fidelity of the quantum state transfer between a superconducting qubit and the ancillary atom as a function of $g t / \pi$. The parameters are $\kappa_{d}=0.1 \mathrm{MHz}, \kappa_{b}=\kappa_{a}=1 \mathrm{MHz}, \gamma_{a}=\gamma_{s}=0.1$ $\mathrm{MHz}$, and $g=6 \pi \mathrm{MHz}$.

After diagonalizing the total Hamiltonian without dissipation, we find that deterministic state transfer is achieved at time $t=\pi / g$, when the relation $2 r^{2}=\left(4 k^{2}-1\right)$ (with $k=1,2,3, \ldots$ and $r=G / g$ ) is fulfilled. To facilitate the state transfer process, larger $G$ is preferable [27]. Meanwhile, to achieve adiabatic transfer of qubits state, $r \gg 1$ is required in order to single out only the dark bosonic mode, which results in much smaller $g$ for a given $G$. As a result, the time needed to complete the transfer will be much longer, and thus decoherence will cause considerable errors. However, if very strong $G$ is experimentally accessible, e.g., $r=20$, which means $G \sim 100 \mathrm{MHz}$, we have numerically confirmed that the influences of mechanical mode decay on the state transfer process can be safely neglected.

Finally, we estimate the influence of dissipation to the state transfer process by integrating the quantum master equation

$$
\begin{array}{r}
\dot{\rho}=-i[H, \rho]+\sum_{\beta} \kappa_{\beta}\left(2 \beta \rho \beta^{\dagger}-\beta^{\dagger} \beta \rho-\rho \beta^{\dagger} \beta\right) \\
+\gamma_{s}\left(2 \sigma_{s}^{-} \rho \sigma_{s}^{+}-\sigma_{s}^{+} \sigma_{s}^{-} \rho-\rho \sigma_{s}^{+} \sigma_{s}^{-}\right) \\
+\gamma_{a}\left(2 \sigma_{a}^{-} \rho \sigma_{a}^{+}-\sigma_{a}^{+} \sigma_{a}^{-} \rho-\rho \sigma_{a}^{+} \sigma_{a}^{-}\right),
\end{array}
$$

where $\rho$ is the density matrix of the entire system, $\beta \in\{a, b, d\}, \kappa_{a}, \kappa_{b}$ and $\kappa_{d}$ are the decay rates of the optical cavity, microwave cavity, and the mechanical oscillator, respectively; $\gamma_{a}$ and $\gamma_{s}$ are the lifetimes of the atomic and superconducting qubits, respectively. We characterize the transfer process for the given initial state by the conditional fidelity of the quantum state defined by $F_{2}=\left\langle\psi_{a}\left|\rho_{a}\right| \psi_{a}\right\rangle$ with $\rho_{a}$ being the atomic reduced density matrix. By choosing the typical parameters: $\kappa_{a}=1 \mathrm{MHz}, \kappa_{b}=1 \mathrm{MHz}$, $\kappa_{d}=0.1 \mathrm{MHz}, \gamma_{a}=0.1 \mathrm{MHz}$ and $\gamma_{s}=0.1 \mathrm{MHz}$, we plot, in Fig. 2, the fidelity of the quantum state transfer process $F_{2}$ as a function of the dimensionless time $g t / \pi$, where we have obtained a high fidelity $F_{2}>95 \%$ of the process. In particularly, even with considerable deviation (about $\pm 10 \%$ ) 
of the coupling strength to the ideal condition, $F_{2}>94 \%$ can still be obtained. Therefore, this process is very robust to the deviation of the coupling strength. In the above estimation, we have neglected the effect from the atoms in the optical lattice due to the following two reasons. Firstly, the cavityassisted interaction can be effectively switched off. Secondly, if there is a small probability that it has not been switched off, then it will cause energy shift of the cavity mode. As for $N$ atoms, this shift is $N g^{2} / \delta \sim N g / 10$ for $\delta \sim 10 g$. For the ancillary atom, we may choose $\delta^{\prime}=10 \Omega_{A}=100 \mathrm{~g}$; in this way $\lambda=0.1 g$ is still large enough for our manipulation purpose. With the above parameters, for $N=5$, we obtain that the atom-induced energy shift is $\delta^{\prime} / 200$, and thus can be safely neglected. Therefore, combining the process of storing the ancillary atomic state into the TQM, we can obtain a fidelity of $F=F_{1} \times F_{2} \approx 86 \%$ for storing a superconducting qubit into the TQM.

\section{CONCLUSION}

In summary, we have proposed a hybrid implementation of a TQM for both atomic and superconducting qubits, which can combine the advantages of both the noise resistance of the topological qubits and the scalability of the superconducting qubits. In particular, by introducing a quantum opto-electromechanical interface, we have demonstrated that the superconducting qubit state can be efficiently transferred into the TSQ.

This work was supported by the NFRPC (Grants No. 2013CB921804 and No. 2012CB921604), the NSFC (Grants No. 11474153, No. 11274069, No. 11474064, NO. 61435007, and NO. 11474177), the PCSIRT (Grant No. IRT1243), and the RGC of Hong Kong (HKU173051/14P and HKU173055/15P).
[1] You J Q, Nori F. Atomic physics and quantum optics using superconducting circuits. Nature, 2011, 474: 589-597

[2] Clarke J, Wilhelm F K. Superconducting quantum bits. Nature, 2008, 453: 1031-1042

[3] Kitaev A. Fault-tolerant quantum computation by anyons. Ann. Phys. (N.Y.), 2003, 303: 2-30

[4] Hasan M Z, Kane C L. Topological insulators. Rev. Mod. Phys., 2010, 82: 3045-3067

[5] Qi X L, Zhang S C. Topological insulators and superconductors. Rev. Mod. Phys., 2011, 83: 1057-1110

[6] Guo H M. A brief review on one-dimensional topological insulators and superconductors. Sci China-Phys Mech Astron, 2016, 59: 637401

[7] Kane C L, Mele E J. $Z_{2}$ topological order and the quantum spin Hall effect. Phys. Rev. Lett., 2005, 95: 146802

[8] Bernevig B A, Hughes T L, Zhang S C. Quantum spin Hall effect and the topological phase transition in HgTe quantum wells. Science, 2006, 314: 1757-1761

[9] Fu L, Kane C L, Mele E J. Topological insulators in three dimensions. Phys. Rev. Lett., 2007, 98: 106803

[10] Moore J E, Balents L. Topological invariants of time-reversalinvariant band structures. Phys. Rev. B, 2007, 75: 121306(R)

[11] Roy R. Topological phases and the quantum spin Hall effect in three dimensions. Phys. Rev. B, 2009, 79: 195322

[12] Ruseckas J, Juzeliūnas G, Öhberg P, Fleischhauer M. NonAbelian gauge potentials for ultracold atoms with degenerate dark states. Phys. Rev. Lett., 2005, 95: 010404

[13] Zhu S L, Fu H, Wu C J, Zhang S C, Duan L M. Spin Hall effects for cold atoms in a light induced gauge potential. Phys. Rev. Lett., 2006, 97: 240401

[14] Zhu S L, Shao L B, Wang Z D, Duan L M. Probing non-abelian statistics of Majorana fermions in ultracold atomic superfluid. Phys. Rev. Lett., 2011, 106: 100404

[15] Lin Y J, Jiménez-García K, Spielman I B. A spin-orbit coupled Bose-Einstein condensate. Nature, 2011, 471: 83-86

[16] Wang P, Yu Z Q, Fu Z, et al. Spin-orbit coupled degenerate Fermi gases. Phys. Rev. Lett., 2012, 109: 095301

[17] Cheuk L W, Sommer A T, Hadzibabic Z, Yefsah T, Bakr W S, Zwierlein M W. Spin-injection spectroscopy of a spin-orbit coupled Fermi gas. Phys. Rev. Lett., 2012, 109: 095302
[18] Zhang J Y, Ji S C, Chen Z, et al. Collective dipole oscillation of a spin-orbit coupled Bose-Einstein condensate. Phys. Rev. Lett., 2012, 109: 115301

[19] Bloch I, Dalibard J, Zwerger W. Many-body physics with ultracold gases. Rev. Mod. Phys., 2008, 80: 885-964

[20] Liu X J, Liu Z X, Cheng M. Manipulating topological edge spins in ome-dimensional optical lattice. Phys. Rev. Lett., 2013, 110: 076401

[21] Jiang L, Brennen G K, Gorshkov A V, et al. Anyonic interferometry and protected memories in atomic spin lattices. Nat. Phys., 2008, 4: 482-488

[22] Wu Z, Zhang L, Sun W, et al. Realization of twodimensional spin-orbit coupling for Bose-Einstein condensates. arXiv: 1512.06394

[23] Kubo Y, Grezes C, Dewes A, et al. Hybrid quantum circuit with a superconducting qubit coupled to a spin ensemble. Phys. Rev. Lett., 2011, 107, 220501

[24] Yang W L, Yin Z Q, Hu Y, Feng M, Du J F. High-fidelity quantum memory using nitrogen-vacancy center ensemble for hybrid quantum computation. Phys. Rev. A, 2011, 84: 010301

[25] Chen Q, Yang W L, Feng M. Controllable quantum state transfer and entanglement generation between distant nitrogenvacancy-center ensembles coupled to superconducting flux qubits. Phys. Rev. A, 2012, 86: 022327

[26] Tao M J, Hua M, Ai Q, Deng F G. Qauntum information processing on nitrogen-vacancy ensembles with the local resonance assisted by circuit QED. Phys. Rev. A, 2015, 91: 062325

[27] Bagci T, Simonsen A, Schmid S, et al. Optical detection of radio waves through a nanomechanical tansducer. Nature, 2014, 507: $81-85$

[28] Andrews R W, Peterson R W, Purdy T P, et al. Bidirectional and efficient conversion between microwave and optical light. Nat. Phys., 2014, 10: 321-326

[29] Yin Z Q, Yang W L, Sun L, Duan L M. Quantum network of superconducting qubits through opto-mechanical interface, Phys. Rev. A, 2015, 91: 012333

[30] Li T, Yin Z Q. Quantum superposition, entanglement, and state teleportation of a microorganism on an electromechanical oscillator. Science Bulletin, 2016 2: 163-171

[31] Xiong H, Si L G, Lv X Y, et al. Review of cavity optomechan- 
ics in the weak-coupling regime: from linearization to intrinsic nonlinear interactions. Sci China-Phys Mech Astron, 2015, 58 : 50302

[32] Schoelkopf R J, Girvin S M. Wiring up quantum systems. Nature, 2008, 451, 664-669

[33] Jiang L, Kane C L, Preskill J. Interface between topological and superconducting qubits. Phys. Rev. Lett., 2011, 106: 130504

[34] Xue Z Y, Shao L B, Hu Y, Zhu S L, Wang Z D. Tunable interfaces for realizing universal quantum computation with topological qubits. Phys. Rev. A, 2013, 88: 024303

[35] Cottet A, Kontos T, Douçot B. Squeezing light with Majorana fermions. Phys. Rev. B, 2013, 88: 195415

[36] Xue Z Y, Gong M, Liu J, Hu Y, Zhu S L, Wang Z D. Robust interface between flying and topological qubits. Sci. Rep., 2015, 5: 12233
[37] Kitaev A Y. Unpaired Majorana fermions in quantum wires. Physics-Uspekhi, 2001, 44: 131-136

[38] Cho J. Addressing individual atoms in optical lattices standingwave driving fields. Phys. Rev. Lett., 2007, 99: 020502

[39] Gorshkov A V, Jiang L, Greiner M, Zoller P, Lukin M D. Conherent quantum optical control with subwavelength resolution. Phys. Rev. Lett., 2008, 100: 093005

[40] Hood C J, Lynn T W, Doherty A C, Parkins A S, Kimble H J. The atom-cavity microscope: Single atoms bound in orbit by single photons. Science, 2000, 287: 1447-1453

[41] Brown K R, Harrow A W, Chuang I L. Arbitrarily accurate composite pulses. Phys. Rev. A, 2004, 70: 052318

[42] Yin Z Q, Li F L. Multiatom and resonant interaction scheme for quantum state transfer and logical gates between two remote cavities via an optical fiber. Phys. Rev. A, 2007, 75: 012324 